\title{
Semi-empirical fission model for r-process based on the recent experiments and three-dimensional Langevin ap- proach
}

\author{
Chikako Ishizuka ${ }^{1,3, *}$, Kohsuke Tsubakihara ${ }^{2}$, Satoshi Chiba $^{1}$, Yuichiro Sekiguchi ${ }^{3}$, and \\ Shinya Wanajo ${ }^{4}$ \\ ${ }^{1}$ Institute of Innovative Research, Tokyo Institute of Technology, Tokyo 152-8550, Japan \\ ${ }^{2}$ National Institute of Technology, Asahikawa College, Asahikawa 071-8142, Japan \\ ${ }^{3}$ Department of Physics, Toho University, Funabashi, Chiba 274-8510, Japan \\ ${ }^{4}$ Max Planck Institute for Gravitational Physics (Albert Einstein Institute), Am Mühlenberg 1, Potsdam- \\ Golm, D-14476, Germany
}

\begin{abstract}
Nuclear fission of superheavy elements can affect the r-process nucleosynthesis via a fission cycling process. In that process, the identification of the nuclide of a fission fragment and its abundance is essential for a precise evaluation of their contribution as seed nuclei of the r-process. We have investigated the nuclear fission of SHEs from the proton-rich side to the neutron-rich side using our three- and four-dimensional Langevin models [1,4]. This model can reproduce the experimental data on nuclear fission of actinides and SHEs, while it can only provide various quantities to each fission fragment, we also developed a semi-empirical charge distribution model based on abundant experimental data of actinides [3]. A new semi-empirical nuclear fission model for the r-process is made by combining these two models.
\end{abstract}

\section{Background and Our Model}

Nuclear fission plays an essential role in the rapid neutron capture process (r-process) because it provides seed nuclei for the r-process. After the first observation of the r-elements in neutron star mergers, the fission cycling process in the r-process attracts more attention for a more precise analysis of the r-process nucleosynthesis. However, fission yields strongly depend on nuclear models. Therefore, we need fission yields based on a theory with predictive power. Our semi-empirical fission fragment model $\mathrm{Y}(\mathrm{Z}, \mathrm{A})$ combines mass yield model Y(A) [2] and the charge distributions evaluated by thousands of experimental data of nuclear fission in actinides [3]. Here, Y(A) [2] is the superposition of three Gaussian functions, which is based on the three-dimensional Langevin calculations with Two Center Shell Model (TCSM) as shown in Figure 1. For details of the charge distributions, please see our previous work [3]. In this paper, we discuss the mass and charge distribution of the present fission model, and evaluate the impact of fission models on the r-process.

$$
\begin{aligned}
Y(Z, A) & \approx Y(A) \\
& \times \frac{1}{\sqrt{2 \pi} \sigma(A)} \int_{-0.5}^{0.5} \exp -\frac{\left(Z-Z_{p}(A)+t\right)^{2}}{2 \sigma(A)^{2}} d t \times \exp -\frac{\Delta E_{s h}(Z, A)}{E_{d}(A)} .
\end{aligned}
$$

*e-mail: ishizuka.c.aa@m.titech.ac.jp 


\section{Potential energy surface with TCSM}

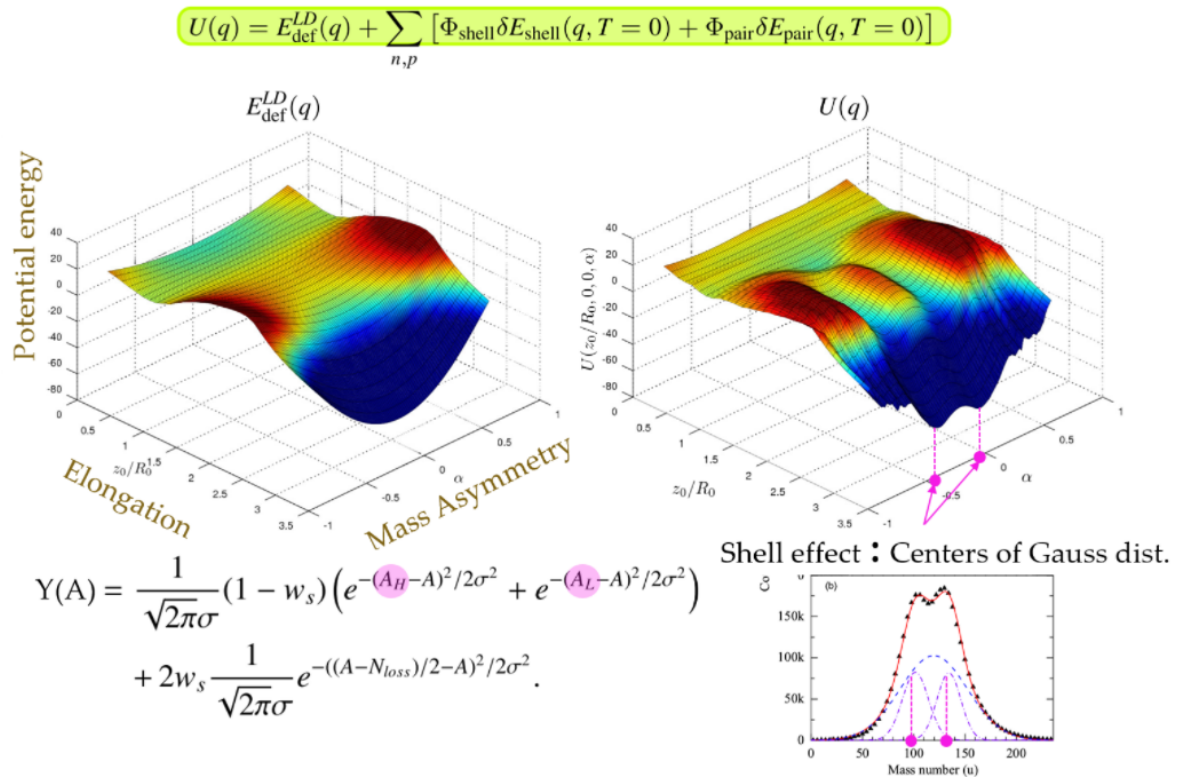

Figure 1. Schematic view of how to construct the mass yield from the potential surface of two center shell model (TCSM). Mass numbers $A_{H}$ and $A_{L}$ in the mass yield $Y(A)$ correspond to the valleys of the potential energy surface with respect to the fission fragment mass number.

\section{Results and Summary}

Figure 2 shows the mass and charge distribution of our $\mathrm{Y}(\mathrm{Z}, \mathrm{A})$ model based on the three dimensional Langevin model [1] with Two Center Shell Model (TCSM). Our model provides a broader mass distribution with the fragment mass number A=90-165 than KodamaTakahashi, the standard mass model of the r-process nucleosynthesis. Especially symmetric mass components are more abundant compared to the standard model. Such a trend can also be seen in the charge distributions, as shown in the lower panel in Figure 2.

In Figure 3, we compare three cases, our Y(Z, A) model, Kodam-Takahashi model, and symmetric fission case, with the mass abundance of the r-process nuclides in the solar system. Using our $\mathrm{Y}(\mathrm{Z}, \mathrm{A})$ model, we obtained an abundance pattern of a single r-process with $Y_{e}=$ 0.05 , corresponding to the electron fraction of a typical black hole and neutron star merger. As a result, we observed significant differences among them, especially around the second peak and the rare-earth peak of the r-process. Thus we found that the fission fragment mass yields can affect the abundance pattern of the r-process via the fission recycling process. Therefore, a more precise fission yield model is necessary to evaluate the contribution of the fission recycling process to the r-process.

For this aim, we have performed the four-dimensional Langevin calculations [4] to more than a hundred nuclides with the proton number $Z=92-122$ from the neutron-deficient side to the neutron-rich side. Figure 3. Compared to the three-dimensional (3D) Langevin model [1], our four-dimensional (4D) Langevin model [4] has an additional degree of freedom concerning nuclear deformation. Therefore, the 4D model can reproduce various nuclear fissions which cannot be well-explained by the 3D model, such as a sudden change 

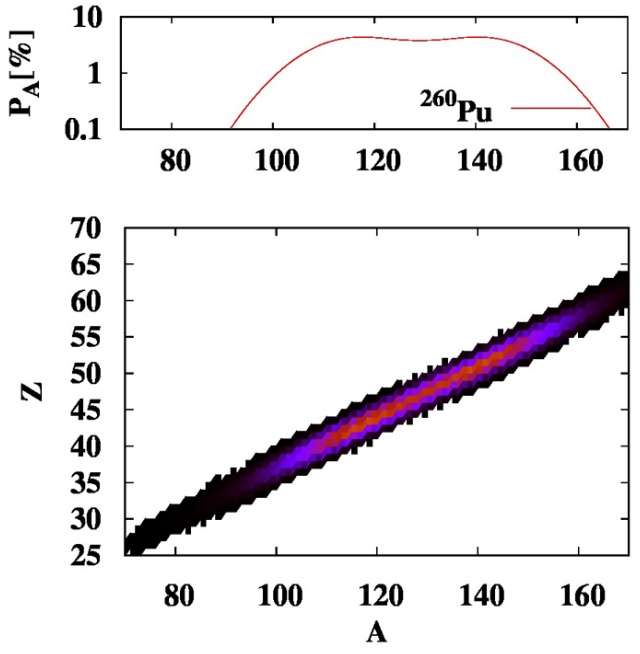

Figure 2. Mass and charge distribution of our semi-empirical yield model $\mathrm{Y}(\mathrm{Z}, \mathrm{A})$ based on three dimensional Langevin model and recent evaluated yield models using abundant experimental data in actinides. The upper panel shows the mass distribution, while the lower color map displays the distributions of fission fragments on a nuclear chart. The center part of the color map is most abundant.

from asymmetric fission to symmetric fission in Fm isotopes. Our new model based on the 4D model will be discussed in a separate paper.

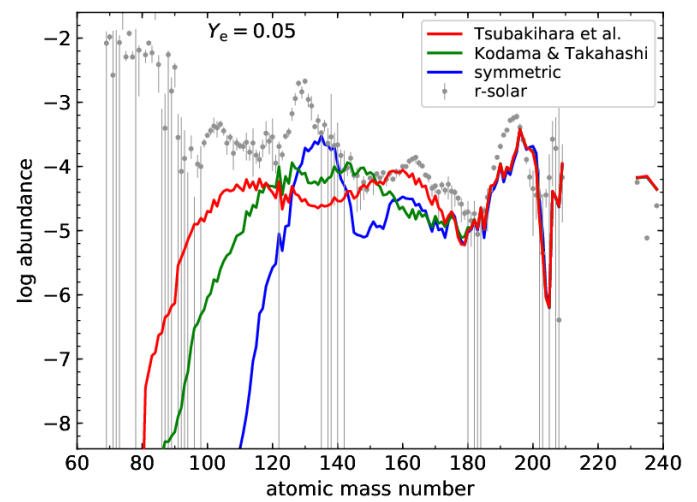

Figure 3. Abundance pattern of a single r-process nucleosynthesis with the electron fraction $Y_{e}=0.05$. Red, green, blue lines are results with present model, Kodama-Takahashi model, and symmetric fission model, respectively. Filled grey circles with error bars are the r-elements in the solar abundance.

\section{Acknowledgement}

This study is supported by Japan Society for the Promotion of Science (JSPS) KAKENHI Grant No. 18K03642.

\section{References}

[1] Y. Aritomo, S. Chiba, Phys. Rev. C 88, 044614,(2013).

[2] M.Ohta et al., Proceedings of "International Conference on Nuclear Data for Science and Technology 2007”, 096 (2008).

[3] K. Tsubakihara, S. Okumura, C. Ishizuka, T. Yoshida, F. Minato, S. Chiba, J. Nucl. Sci. Technol. 58, 151 (2020).

[4] C. Ishizuka, M.D. Usang, F.A. Ivanyuk, J.A. Maruhn, K. Nishio, S. Chiba, Phys. Rev. C 96, 064616 (2017). 